\title{
L'orthographe dans le recrutement : critère implicite de sélection à l'embauche des jeunes
}

Loïc Drouallière

\section{(2) OpenEdition}

1 Journals

Édition électronique

URL : http://journals.openedition.org/communicationorganisation/4822

DOI : 10.4000/communicationorganisation.4822

ISBN : : 979-10-300-0155-6

ISSN : 1775-3546

Éditeur

Presses universitaires de Bordeaux

Édition imprimée

Date de publication : 1 décembre 2014

Pagination : 279-292

ISBN : 978-2-86781-905-6

ISSN : $1168-5549$

\section{Référence électronique}

Loïc Drouallière, «L'orthographe dans le recrutement : critère implicite de sélection à l'embauche des jeunes », Communication et organisation [En ligne], 46 | 2014, mis en ligne le 01 décembre 2017, consulté le 26 avril 2019. URL : http://journals.openedition.org/communicationorganisation/4822 ; DOI : 10.4000/communicationorganisation.4822 


\title{
L'orthographe dans le recrutement : critère implicite de sélection à l'embauche des jeunes
}

\author{
Loïc Drouallière ${ }^{1}$
}

\section{Introduction}

L'orthographe fait depuis longtemps l'objet de nombreuses recherches en matière d'éducation et de linguistique : son apprentissage, son sens, son histoire, ses remédiations. Si ses enjeux sociaux ont été mis en avant en tant que convention sociale (Bourdieu, 1979) ou en tant que norme prescriptive et évaluative (Kasbarian, Baggioni et Grimaldi,1994), l'étude de ses conséquences professionnelles (Millet \& Billiez, 1994) reste plus discrète. Dejonghe et al. (1996), Manesse et Cogis (2007) puis Drouallière (2013) ont tour à tour démontré que la baisse moyenne du niveau de maîtrise orthographique frappe les apprenants du collège à l'université. En revanche, les conséquences en termes de vie professionnelle et sociale sont difficiles à établir. Pour ce qui touche à l'insertion professionnelle, le candidat à un emploi dévoilera peu ou prou sa maitrise orthographique lors des communications normées que sont le curriculum vitæ et la lettre de candidature. Ici, l'intérêt est de découvrir si une orthographe lacunaire expose un jeune diplômé à une disqualification à l'embauche à l'occasion d'un premier ou deuxième emploi.

Si l'acte d'évaluation des compétences d'un candidat relève de la psychologie sociale de par les normes sociales de jugement qu'il sollicite, la candidature normée en réponse à une offre d'emploi est avant tout un acte et une situation de communication. L'interprétation d'une orthographe lacunaire par un spécialiste du recrutement repose sur des comportements et des valeurs qu'il attribue dans l'absolu au profil du poste : bases non acquises, carences motivationnelles, négligence, naïveté. Le message est porteur de significations appelées à être décodées puis analysées par le destinataire (Watzlawick et al., 1972). Par extension, le spécialiste du recrutement tente de traduire ce manquement épistolaire en termes d'efficacité professionnelle. Les futurs dossiers seront-ils traités avec la même négligence ? Les futures

1 Loïc Drouallière est docteur en Sciences de l'éducation et chercheur en Sciences de l'information et de la communication. Il est rattaché au laboratoire I3M de l’Université de Toulon ; loic.droualliere@univ-tln.fr 
responsabilités s'accommoderont-elles d'une part de naïveté ? Les futurs doutes généreront-ils une recherche d'informations ? Il est probable que l'importance qu'accorde un spécialiste du recrutement à la compétence orthographique et, plus largement, à la littératie soit fonction du type de poste envisagé et du standing de la prestation commercialisée.

Dans la concurrence économique actuelle, l'organisation est de plus en plus soucieuse de l'image perçue qu'elle donne à ses acteurs intérieurs et extérieurs. Si les collaborateurs en sont les vecteurs au travers de leurs productions matérielles et immatérielles, la crédibilité de l'organisation a aussi de quoi se fonder sur la qualité de l'orthographe des écrits professionnels qui circulent en son sein et en émanent (Hotier, 1996). Le souci de préserver cette crédibilité pourrait inciter les recruteurs à discriminer les candidatures dont les supports sont pourvus d'erreurs orthographiques. Aucune étude ne s'est jamais, à notre connaissance, souciée de vérifier et mesurer une prétendue sélection à l'embauche. Après avoir explicité le cadre théorique nécessairement pluriel de ce protocole de sélection orthographique à l'embauche, nous présenterons la méthode utilisée afin de duper les recruteurs, les caractéristiques des profils des candidats fictifs et le recueil des données. Les résultats de l'expérimentation précéderont des éléments d'interprétation ainsi qu'une réflexion sur les perspectives ainsi ouvertes et les conséquences professionnelles.

\section{Cadre théorique}

Il ne s'agit pas ici d'appréhender l'orthographe purement comme un code linguistique, agrégat de normes techniques, mais bien comme un code social vecteur de communications internes et externes à l'organisation. Au-delà de la norme prescriptive, Wynants (1997) définit le code graphique comme une norme évaluative dont le principe est d'attribuer une valeur esthétique ou morale aux formes linguistiques. À partir de cette norme, se pose la question de l'influence exercée sur le destinataire par les erreurs contenues dans l'énoncé qu'il lit ; une influence qui ne dépend pas de l'intention signifiante. On partira du principe qu'un énoncé parsemé de nombreuses variations du code graphique est un acte non intentionnel. Par conséquent, il s'agit ici de considérer l'acte perlocutoire de l'énoncé (Austin, 1970) ; l'émetteur d'une communication écrite ignore l'effet psychologique produit par les variations formelles de son énoncé - en tant qu'écarts à la norme graphique - sur le destinataire.

Dans le milieu professionnel, l'orthographe constitue, malgré les évolutions socioculturelles qu'elle subit, une préoccupation vivace liée à l'image sociale que véhiculent les écrits dont les différents membres de l'organisation en sont auteurs. Ainsi, l'orthographe octroie au professionnel une forme de crédibilité qui peut s'amalgamer à sa compétence technique et concrète et, par extension, à celle de l'organisme qu'il représente (Korchia, 2000, Maccioni, 2010). Dans le cas de l'orthographe en tant que convention, une information graphiquement 
codée se fait parasiter par des variations par rapport à la norme avant même d'emprunter le canal en direction du destinataire. Ces variations créent une «saillance linguistique due à une mise en avant explicite » d'une forme sur un fond, d'une irrégularité sur un contexte uniforme (Landragin, 2004 cité par Leleu-Merviel et Useille, 2008).

Toute sélection ou évaluation se fonde sur des normes sociales de jugement qui permettront de différencier implicitement les événements en fonction de leur désirabilité du point de vue du groupe qui génère la norme. Dans le cas d'une réponse à une offre d'emploi, le candidat tente de maximiser sa désirabilité sociale auprès du lecteur de ses documents de candidature, de manière à déclencher chez celui-ci un comportement sélectif favorable. C'est sans doute l'une des situations d'écriture les plus formelles car elle émane d'un rapport hiérarchique entre scripteur et destinataire. Le premier se soumet à l'évaluation du second et cherche à se montrer à son avantage. Le recrutement est un phénomène social et culturel complexe où se nouent des enjeux de pouvoir aux conséquences importantes sur le plan économique, pour l'entreprise, et sur le plan personnel, pour le candidat. Consécutivement, Eymard-Duvernay et Marchal (1997) définissent la notion de conventions de compétences qui s'appliquent de manières très diverses lors d'un recrutement et varient selon la position hiérarchique ou la fonction occupée au sein de l'organisation. La compétence orthographique pourrait faire partie de ces conventions souvent spécifiques au poste, au secteur d'activité et/ou à l'organisation.

Le recrutement relève sans doute possible des sciences de gestion en tant que processus des ressources humaines. Néanmoins, cette recherche porte précisément sur un acte de langage écrit s'inscrivant dans un processus de recrutement; il s'agit par conséquent d'une communication organisationnelle. Ce processus se caractérise par une situation de communication asynchrone formalisée dont on peut diviser le processus communicationnel en deux étapes:

1. La première étape de cette situation réside dans la parution de l'offre d'emploi émise par l'entreprise. En amont, l'employeur cherche à rappeler aux interlocuteurs potentiels ses statut et pouvoir hiérarchiques en leur imposant un contrat de communication (Ghiglione, 1986) par lequel il donne sa définition de la situation de communication (profil exigé, modalités de recrutement, canaux de réception des candidatures).

2. Quelques heures, quelques jours plus tard, la réponse du candidat à cette offre d'emploi constitue la deuxième étape de la situation. Après avoir réuni les conditions qu'il estime nécessaires pour mettre en mots, il rédige ses documents de candidature en respectant les conventions formelles lues ou enseignées.

Obligé de communiquer s'il veut obtenir ce qu'il souhaite, le sujet est contraint à se lancer dans un échange langagier qui, par définition, l'expose 
à son destinataire. Si le candidat est logiquement demandeur d'un jugement qu'il espère favorable, il s'expose tout autant à un refus, davantage perçu comme une négation de ses compétences que comme le privilège accordé à une candidature plus en adéquation avec les attentes de l'organisation. Au-delà des stratégies de discours (Charaudeau, 1995) employées par le candidat afin d'augmenter sa désirabilité sociale (Beauvois, 1976), subsiste une communication fonctionnelle (Wolton, 1997), directement utile, à travers les formes que revêt la candidature : mise en page et photographie choisies, polices de caractère utilisées, variations orthographiques. À cette distinction près que les éventuelles erreurs d'orthographe ne représentent pas un choix délibéré de la part du candidat. Pour Watzlawick et al. (1972), toute communication contient une double information; d'une part, sur le contenu du message et, d'autre part, sur la manière dont ce message est émis. La communication a notamment pour fonction de développer, maintenir, entretenir l'image positive de soi que l'on tente de présenter aux autres (Goffman, 1973), que ce soit à travers le langage, les postures, l'habillement. En l'absence du candidat, le curriculum vitæ endosse le rôle de messager : celui qui va présenter et représenter le candidat au recruteur, celui qui est chargé d'en véhiculer l'image positive la plus proche de l'idéal que s'en fait le recruteur.

\section{Méthode}

Mesurer une sélection à l'embauche ne peut reposer que sur une recherche expérimentale de terrain, dans la mesure où les participants sont des habitués du contexte où a lieu l'expérimentation qui les piège à leur insu. Ici, nous formulons l'hypothèse que les erreurs orthographiques des documents de candidature agissent comme critère implicite de sélection préjudiciable à la désirabilité recherchée par le candidat. Pour tester celle-ci, nous avons mis au point un dispositif méthodologique susceptible de provoquer un jugement sélectif spontané en conformité avec une norme sociale implicitement partagée. Dans une logique bypothético-déductive, nous le concevons en faisant appel à ces mêmes normes qui construisent chez le sujet une exigence en matière de code graphique à l'origine d'un comportement évaluatif. Effectué en milieu naturel, ce plan d'expérience à mesures répétées consiste à " confronter un seul et même groupe de sujets à tous les niveaux de la variable indépendante » (Courbet, 2010). Il vise à comparer les prises de contact initiées par les recruteurs entre deux profils de candidat similaires, le premier à l'orthographe impeccable, le second à l'orthographe lacunaire.

Dans ce cadre, nous prêtons une attention toute particulière aux profils qui font l'objet de ces candidatures fictives. Ceux-ci doivent être rigoureusement similaires sur le fond afin de ne pas biaiser les résultats; nous nous attachons à mesurer la seule influence de la correction orthographique sur la perception sélective du recruteur. Cela suppose de neutraliser le maximum d'éléments formels liés à toute candidature de ce type (consonance patronymique, 
domiciliation socio-topographique, situation de famille, âge, caractéristiques faciales de la photographie, message d'accueil sur la messagerie téléphonique, possession du permis de conduire...). Le recruteur mobilise ainsi ses normes de jugement basées sur les exigences professionnelles de son secteur d'activité et/ou de l'organisation à laquelle il appartient ainsi que sur la valeur intrinsèque qu'il accorde à la correction orthographique. L'objectif est ici d'isoler au maximum la variable orthographique afin de mesurer, toutes choses égales par ailleurs, l'influence qu'elle exerce en tant que critère de sélection dans le processus de recrutement.

\section{Variables indépendante et dépendante}

- V.i. : erreurs orthographiques dans les documents de candidature (trois modalités)

- V.d. : prises de contact initiées par les recruteurs sollicités

\begin{tabular}{|c|c|c|c|} 
Hypothèses & $\begin{array}{c}\text { CV0 } \\
+ \text { LC0 }\end{array}$ & $\begin{array}{c}\text { CV4 } \\
+ \text { LC10 }\end{array}$ & $\begin{array}{c}\text { CV9 } \\
+ \text { LC10 }\end{array}$ \\
\hline $\mathrm{H} 1$ & $\checkmark$ & \multicolumn{2}{|c|}{} \\
\hline $\mathrm{H} 2$ & $\checkmark$ & $\checkmark$ & \\
\hline $\mathrm{H} 3$ & $\checkmark$ & & $\checkmark$ \\
\hline
\end{tabular}

CV4 / CV9 : 4 puis 9 erreurs d'orthographe contenues dans le CV. LC10 : 10 erreurs contenues dans la lettre de candidature.

La mesure des résultats porte sur les prises de contact consécutives à l'envoi simultané des candidatures jumelles. Nous considérons qu'il y a prise de contact à partir du moment où le candidat est sollicité par le recruteur, soit pour se rendre à un entretien, soit pour donner des informations complémentaires. À l'inverse, nous considérons la réponse comme négative si le chargé de recrutement rejette formellement la candidature ou s'il n'y répond pas.

\section{Constitution de l'échantillon}

L'échantillon est constitué d'offres d'emploi qui visent à pourvoir des postes de conseillers commerciaux. Les annonces sont sélectionnées sur des sites internet, soit spécialisés en offres d'emploi, soit d'entreprises dans leur rubrique "Recrutement ", répondant ainsi à des demandes concrètes et immédiates. Pour maximiser les prises de contact potentielles, les deux profils de candidat tentent d'être en adéquation maximale avec les éléments d’information contenus dans le libellé de l'annonce. 


\section{Caractéristiques des profils fictifs de commerciaux junior}

Les candidatures jumelles s'effectuent en ligne, directement sur le site web de l'organisation qui recrute, via un formulaire plus ou moins exhaustif que le candidat complète en plusieurs étapes (état civil, coordonnées, formation, expériences professionnelles, compétences diverses...). Au terme de quoi, le candidat doit télécharger deux fichiers texte correspondant à son curriculum vitæ et à sa lettre de candidature. Dans les documents du candidat à l'orthographe lacunaire, les erreurs orthographiques et grammaticales sont soigneusement réparties par natures d'erreurs sur l'ensemble des deux documents de candidature. Afin de susciter une saturation et déterminer un seuil de tolérance, nous optons pour deux conditions expérimentales concernant le nombre d'erreurs (4 puis 9) contenues dans le CV; le nombre d'erreurs (10) de la lettre de candidature reste lui inchangé.

\section{Modalités expérimentales}

La validité d'une offre dépend de sa réponse aux caractéristiques suivantes : secteur d'activité sélectionné, poste à vocation commerciale, expérience exigée du candidat entre une et cinq années, contrat de travail à durée indéterminée. Une fois l'offre validée, débute la rédaction des documents de candidature. Pour les curriculum vitæ, les éléments formels sont systématiquement neutralisés. Quant aux éléments non formels, nous veillons à ce qu'ils n'éveillent pas les soupçons du lecteur destinataire. Les deux fichiers qui accompagnent la candidature en ligne portent une extension informatique différente ; lintitulé des fichiers est libellé différemment pour éviter toute confusion entre les deux candidats. L'envoi des documents de candidature ne s'effectue pas de manière simultanée mais en léger différé ; l'un avant minuit, l'autre en tout début de matinée. Ainsi, l'on peut considérer que le chargé de recrutement destinataire se saisira des deux dossiers de candidature au même instant.

Les données sont recueillies, soit par courrier électronique reçu sur la messagerie électronique propre à chacun des deux candidats fictifs, soit par appel téléphonique reçu sur la ligne de téléphonie mobile dédiée à chacun d'eux, qu'il y ait un message déposé par le chargé de recrutement ou simplement l'affichage du numéro de l'appelant (en cas d'appel sans message).

L'intérêt principal de ce protocole expérimental réside dans le fait de savoir s'ily a prise de contact ou non de la part des lecteurs destinataires des documents de candidature, selon la présence ou l'absence d'erreurs orthographiques dans ces derniers. Par conséquent, toute prise de contact initiée par le recruteur, en particulier par le vecteur courriel, nécessite évidemment de juger si la formulation utilisée évoque une fin de non-recevoir ou bien une volonté de poursuivre le processus de recrutement. Les réponses positives intègrent sans distinction : souhait d'entretien téléphonique, proposition d'entretien d'embauche physique, demande d'informations complémentaires. De la même manière qu'aucune relance n'est rendue possible en l'absence de 
moyens de contact avec le/les chargé(s) de recrutement, aucun désistement n'est prévu de la part du candidat qui serait contacté en premier. Il n'y aura aucun contact ultérieur (du ou des candidats vers l'entreprise ciblée) au dépôt de la candidature, même en cas de demande d'informations, de sollicitation téléphonique, voire de relance(s). Dans l'ensemble, ce sont 189 offres d'emploi auxquelles fut envoyée une double candidature. Dans les deux modalités expérimentales, le nombre d'erreurs contenues dans la lettre de candidature est, lui, resté inchangé.

\section{Résultats consécutifs aux doubles candidatures de commerciaux junior}

$\mathrm{Au}$ cours de ces résultats, nous verrons dans quelle mesure l'orthographe intervient comme critère de sélection à l'embauche. Nous analyserons, par la suite, l'influence exercée par la taille de l'entreprise sollicitée ainsi que le secteur d'activité concerné.

\section{L'orthographe en tant que critère implicite de sélection à l'embauche}

Le principal objectif de ce protocole en milieu naturel était de savoir si les jugements normatifs des recruteurs candides allaient privilégier les candidatures à l'orthographe impeccable par rapport aux candidatures à l'orthographe lacunaire, de savoir si l'orthographe constitue une compétence distinctive entre deux profils professionnels similaires. Le résultat est sans appel : les chargés de recrutement privilégient fortement les candidatures à l'orthographe impeccable $\left(\mathrm{khi}^{2}=10,87, \mathrm{ddl}=1, \mathrm{p}<.001\right)$. Concrètement, un candidat junior à l'orthographe irréprochable augmente de $56 \%$ ses prises de contact lorsqu'il postule à une offre d'emploi de commercial. De cette manière, c'est bien l'image positive de soi (Goffman, 1973) qu'il augmente ainsi.

En matière d'emploi, la discrimination ${ }^{2}$ se définit comme le traitement spécifique défavorable d'un individu jugé sur un attribut non productif alors qu'il présente les mêmes caractéristiques productives qu'un autre individu traité favorablement. En principe, la discrimination relève de la catégorisation sociale (les femmes, les Noirs, les handicapés, les seniors...) et génère des comportements ségrégatifs à l'égard des membres d'un exogroupe vis-à-vis duquel nous entretenons des préjugés. Or, nous sommes ici en présence d'une discrimination qui ne répond pas à ce principe, sauf à considérer que les mauvais « orthographeurs" constitueraient un exogroupe (Bourdieu, 1979). Nous qualifions de discrimination implicite de compétence la sélection opérée par un recruteur sur un attribut censément non-productif (puisqu'absent du libellé de l'offre) mais finalement considéré comme productif lors de l'analyse des documents de candidature. Néanmoins, les résultats de cette recherche ne

2 L'attention du lecteur est attirée sur le sens accordé au terme discrimination. Il s'agit ici de l'acception littérale qui signifie «Action de séparer, de distinguer deux ou plusieurs êtres à partir de certains [...] caractères distinctifs » (Larousse, 2014) et non pas « le fait de séparer un groupe social des autres en le traitant plus mal ». 
permettent de savoir ni dans quelle mesure ni de quelle manière les recruteurs considèrent la compétence orthographique comme un attribut productif.

Lors d'une réponse à une offre d'emploi, le candidat ne se contente pas d'envoyer son curriculum vitae ; il émet un ensemble de signes dont il ne contrôle pas la perception par le recruteur ni « la manière dont ces signaux sont effectivement décodés selon les prérequis du poste à pourvoir » (Petit, Duguet, L'Horty, Du Parquet et Sari, 2010). Le candidat ne connaît que le résultat dichotomique de cette analyse : c'est positif ou négatif. Privé d'un feed-back salutaire, le candidat ignore très majoritairement la/les raison(s) de sa disqualification, en fonction de laquelle/desquelles il peut rediriger, modifier, améliorer sa démarche de recherche d'emploi, tant sur le fond que sur la forme. Dans leurs tests, Petit et al. (2010) qualifient de discriminatoire l'attribution arbitraire d'une valeur professionnelle à une caractéristique non professionnelle telle que le sexe, le lieu de résidence et l'origine ethnique. En ce qui concerne notre recherche, les résultats expérimentaux ne relèvent pas d'une telle attribution. Par conséquent, si la maîtrise orthographique constitue un stéréotype mobilisé par les recruteurs, cela fait d'elle une valeur professionnelle, au même titre que les compétences techniques constitutives d'un curriculum vitae. Or, il est tout à fait concevable de penser que des candidats, écartés d'une sélection pour cause d'orthographe lacunaire, invoqueraient une discrimination à l'embauche, arguant qu'un tel critère peut s'appliquer pour des journalistes mais pas pour des commerciaux. En l'espèce, Rotter (1954) souligne qu'un individu aura du mal à attribuer une causalité interne aux événements défavorables qu'il subit.

Il existe une différence notable dans l'effet produit par un curriculum vitae parsemé de 4 erreurs d'orthographe $\left(\chi^{2}=2,11 ; \mathrm{ddl}=1 ; \mathrm{p}<.1\right)$ et le même agrémenté de 9 erreurs $\left(\chi^{2}=14,00 ; \mathrm{ddl}=1 ; \mathrm{p}<.001\right)$. Une candidature à l'orthographe impeccable double les sollicitations de la part des entreprises ciblées (48 contre 24 prises de contact). Un tel écart au test d'indépendance laisse à penser qu'il existe un seuil de tolérance, situé entre 5 et 8 erreurs d'orthographe, au-delà duquel les recruteurs seraient sensibles à ce critère de sélection et opéreraient une distinction entre les candidats. On se rend bien compte que la sélection de candidatures en vue d'une possible embauche relève d'une procédure de jugement normatif, au même titre qu'une évaluation scolaire. Ces résultats ne sont pas sans rappeler ce qu'Yvan Abernot (1988) définit comme variables de débordement prises en compte par le correcteur enseignant, sans qu'il en prenne toujours conscience, dès que leur nombre dépasse un certain seuil, relevant soit du critère d'évaluation, soit de l'exaspération. La plupart du temps, « ces variables de débordement ne sont pas comptabilisées quand elles sont rares et peuvent faire baisser la note lorsqu'elles dépassent certaines limites » (Abernot, 1988). Si l'en était besoin, cela confirme que la correction orthographique est bien une norme sociale et, à ce titre, agit en tant que telle lors d'un processus de sélection. 
En outre, et bien que ce ne fût pas l'objet de cette expérimentation, cet écart fait apparaître le curriculum vitae comme le document central de toute candidature traditionnelle. Les recruteurs semblent lui accorder une attention prioritaire, au détriment de la lettre de candidature. Dans la mesure où cette dernière comportait dix erreurs d'orthographe dans les deux conditions expérimentales, on peut en déduire que les recruteurs ont tendance, soit à la survoler de sorte qu'ils ne remarquent pas ou peu les lacunes qu'elle contient, soit à l'ignorer purement et simplement.

\section{Critère variable selon la taille de l'entreprise}

La taille de l'entreprise constitue un critère manifeste dans le fait de sélectionner ou non une candidature selon la qualité de son orthographe. Les grandes entreprises y sont très sensibles $\left(\chi^{2}=7,05 ; \mathrm{ddl}=1 ; \mathrm{p}<.01\right)$ tandis que les PME le sont moins $\left(\chi^{2}=3,41 ; \mathrm{ddl}=1 ; \mathrm{p}<1\right)$ et les TPE encore moins $\left(\chi^{2}=0,42 ; \mathrm{ddl}=1 ; \mathrm{p}<.1\right)$. Respectivement, les sollicitations envers les candidatures à l'orthographe impeccable sont supérieures de $77 \%$ pour les grandes entreprises et de $57 \%$ pour les entreprises de moins de 20 salariés. Autrement dit, plus l'entreprise est grande, plus elle prête attention à l'orthographe des candidats à l'embauche. Sans doute, l'image perçue des grandes entreprises est-elle plus prégnante lorsque leur notoriété les expose médiatiquement à tout discrédit vis-à-vis des agents économiques. Autant les juristes peinent à évaluer le manque à gagner du fait de l'atteinte à l'image de marque par un agent externe (Nussenbaum, 1993), autant cette atteinte devient encore plus abstraite quand elle émane d'un agent interne (Isgour, 2007). Par extension, l'auteur d'erreurs orthographiques subit un opprobre relatif dans la vie sociale lié à ses lacunes. Ce même auteur, placé dans un environnement où ses relations professionnelles seraient amenées à apprécier ou déprécier l'entreprise dont il est un représentant en fonction de l'image qu'il en véhicule, ferait subir à celle-ci un opprobre préjudiciable à son image sociale et à sa compétence professionnelle globale. Une autre analyse consiste à estimer que le panel de candidats à l'embauche est largement plus conséquent dans les grandes entreprises que dans les PME. Ainsi, l'orthographe pourrait constituer un critère de sélection indirect quand les critères traditionnels (expériences, diplôme) ne suffisent à opérer une sélection. Mais il pourrait bien opérer dès le début du processus évaluatif, avant même que les critères traditionnels n'entrent en ligne de compte, grâce à un processus de saillance linguistique à la fois visuel et cognitif (Landragin, 2004).

\section{Des secteurs d'activité diversement concernés}

Nous avons adressé des candidatures fictives dans plusieurs secteurs d'activité commerciale afin de mesurer les éventuelles différences de sélection. Tandis que les négociateurs immobiliers $\left(\chi^{2}=0,34 ; \mathrm{ddl}=1 ; \mathrm{p}<.1\right)$ ne semblent pas ou peu écartés en raison de leurs lacunes orthographiques, les vendeurs 
automobiles $\left(\chi^{2}=4,29 ; \mathrm{ddl}=1 ; \mathrm{p}<.05\right)$, les conseillers en assurance $\left(\chi^{2}=\right.$ $5,61 ; \mathrm{ddl}=1 ; \mathrm{p}<.02)$ et les conseillers financiers $\left(\chi^{2}=5,50 ; \mathrm{ddl}=1 ; \mathrm{p}<.02\right)$ le sont fortement lorsque leur curriculum vitae recèle 9 erreurs.

\section{Validité}

$\mathrm{Au}$ niveau de la validité externe, notre expérience semble répondre aux critères de validité écologique en raison de son caractère « naturel »; elle tente de mettre en relation les caractéristiques socio-professionnelles de la situation d'évaluation dans laquelle se trouvent les recruteurs avec les conduites aux réponses qu'ils produisent dans cette situation. Les prises de contact obtenues pour l'un et l'autre des candidats fictifs attestent du réalisme des documents de candidature. En ce qui concerne sa validité interne, les caractéristiques du test de discrimination nous ont préservé de toute intervention intentionnelle ou non dans le processus méthodologique.

Étant donné notre focalisation sur les profils commerciaux et notre restriction à quatre secteurs d'activité (automobile, immobilier, assurance, banque) parmi les plus émetteurs d'offres d'emploi, il serait hasardeux de généraliser les résultats obtenus sur les commerciaux à d'autres corps de métiers (non cadres) dont la nature expose plus discrètement les écrits qu'ils envoient en externe. Nous sommes conscient que des variables externes telles que le sexe de l'évaluateur, la pression hiérarchique, les normes propres au recrutement dans chaque entreprise, l'ancienneté dans l'entreprise, l'expérience dans le métier, le secteur d'activité, la profusion des candidatures, le délai imparti pour la sélection, la taille de l'entreprise... ont pu agir à notre insu sur les résultats de notre recherche.

\section{Pistes de réflexion}

À l'issue de la présentation des résultats, nous orientons notre réflexion dans trois directions : le caractère implicitement productif de la compétence orthographique, les motivations des organisations à appliquer cette sélection implicite et enfin la justice organisationnelle.

\section{L'orthographe en tant qu'attribut productif implicite}

En milieu professionnel, l'orthographe rentre dans une compétence globale, dite " rédactionnelle », dont les attentes organisationnelles en termes de productivité restent souvent tues. En effet, la grande majorité des libellés des offres d'emploi auxquelles nous avons répondu n'évoquaient jamais la moindre compétence rédactionnelle attendue du candidat. Si l'organisation ne communique pas clairement sur cette compétence précise, c'est qu'elle la considère comme compétence implicite, s'apparentant à l'idée de convention de compétences développée par Eymard-Duvernay et Marchal (1997). L'organisation attend du salarié, selon son rôle et son statut, de produire certains types d'écrits et l'évaluera «en fonction de sa capacité à reproduire 
des normes scripturales implicites » (Fraenkel, 2001) propres à l'organisation, à son secteur d'activité. En ce sens, la littératie et l'orthographe s'affichent comme un attribut productif du salarié. Au sein d'une équipe, le collaborateur le plus à l'aise avec les compétences rédactionnelles sera plus souvent promu. Mais, en phase de recrutement comme en phase de promotion interne, la compétence rédactionnelle reste souvent implicite.

\section{Motivations des organisations à discriminer}

Les résultats de notre étude font état d'une sélection à l'embauche en partie basée sur le critère orthographique. Néanmoins, nous ignorons si ce critère était rédhibitoire chez les recruteurs dupés ou s'il intervenait en second lieu, après la comparaison des profils des candidats jumeaux mais aussi des candidats tiers ayant répondu à l'annonce. De même, nous ne savons rien des motivations des recruteurs et/ou des entreprises à appliquer ce critère; nous ne pouvons qu'émettre des conjectures :

1. Face à l'ampleur des candidatures, le recruteur pourrait choisir un critère arbitraire, en l'occurrence l'orthographe, qu'il soit considéré ou non comme attribut productif au sein de son organisation.

2. La sélection implicite par l'orthographe pourrait répondre à des directives, imposées aux chargés de recrutement, en cohérence avec une stratégie de communication corporate globale.

3. A travers le recrutement, l'organisation privilégierait, implicitement ou non, des candidats au capital linguistique supérieur qu'elle interprète comme un adjuvant de la promotion interne.

\section{Le concept de justice organisationnelle}

Les résultats mis en lumière grâce à cette expérimentation in situ rentrent dans le cadre de la réflexion ouverte par un concept situé à la croisée de la communication, du management et de la psychologie sociale : la justice organisationnelle. Celle-ci s'intéresse aux perceptions des salariés face aux pratiques et politiques des entreprises et des organisations où ils travaillent. Dirk Steiner (2000) a développé des recherches sur la perception de la justice procédurale dans plusieurs méthodes de recrutement. Il apparaît qu'est perçue comme juste une méthode qui paraît logique par rapport à l'emploi visé et qui offre au candidat la possibilité de montrer ses points forts. Or, du point de vue du candidat écarté, à quel motif attribuerait-il le rejet de sa candidature s'il apprenait avoir été écarté pour excès d'erreurs orthographiques dans son curriculum vitae ? Parviendrait-il à concevoir qu'il s'agit d'une compétence légitimement requise par son employeur, au même titre que les autres compétences techniques qu'il affiche ? À l'inverse, estimerait-il dans un tel cas de figure pâtir d'un préjugé anachronique ou abusif ? 


\section{Conclusion}

$\mathrm{Au}$ terme de cette expérience portant sur l'insertion professionnelle des juniors, il apparaît clairement que les recruteurs exercent une sélection à l'embauche basée, entre autres, sur le critère orthographique. Celle-ci est si prégnante qu'on peut difficilement la nier ou la minorer. Si elle ne concerne pas que les jeunes diplômés, il semble que ce soit une population particulièrement exposée. D'une part, parce qu'ils appartiennent à une génération dont trois études attestent du recul de la maîtrise orthographique ; d'autre part, parce que leur faible expérience ne prédispose pas les recruteurs à s'intéresser d'emblée à leur profil professionnel.

D'un point de vue organisationnel, la compétence orthographique se présente comme un attribut productif implicite du candidat. En ce sens, Bougnoux et Bourdin (2001) notent " que l'informatique en réseau et les échanges en temps réel réhabilitent l'orthographe comme critère professionnel discriminant, de manière formelle au sein des DRH dans le cadre des définitions de profil dans le recrutement [...] ». Or, cette compétence reste étrangement absente des libellés des offres d'emploi. En tant que vecteur graphique des communications écrites internes et externes, l'organisation pourrait bien considérer l'orthographe comme un élément de la qualité totale mais se garde toutefois de communiquer clairement.

\section{BIBLIOGRAPHIE}

ABERNOT Y., Les méthodes d'évaluation scolaire, Paris, Dunod, 1996.

AUSTIN J.-L., Quand dire c'est faire, Paris, Seuil, 1970.

BEAUVOIS J.-L., " Problématique des conduites sociales d'évaluation », Connexions, 1976, 19.

BOUGNOUX D. et BOURDIN S., " L'éclairage médiologique pour penser les organisations », Communication E Organisation, 2001, 19.

BOURDIEU P., La distinction : critique sociale du jugement, Paris, Éditions de Minuit, 1979.

BOURDIEU P., Ce que parler veut dire : l'économie des échanges linguistiques, Paris, Fayard, 1982.

CHARAUDEAU P., "Ce que communiquer veut dire », Revue des Sciences Humaines, 1995, 51.

COURBET D., Objectiver l'humain? : volume 2, communication et expérimentation, Paris, Hermès Science Publications, 2010.

DEJONGHE V., LEVASSEUR J., ALINAUD B., PERETTI C., PETRONE J.C., PONS C., THÉLOT C., "Connaissances en français et en calcul des élèves des années vingt et d'aujourd'hui : comparaisons à partir des épreuves du certificat d'études primaires ", Les Dossiers d'Éducation et Formations, 1996, 62. 
DROUALLIÈRE L., Le niveau de maîtrise orthographique du français chez les étudiants de première année universitaire : évolution, enjeux, remédiation, thèse de doctorat, AixMarseille Université, mars 2013.

EYMARD-DUVERNAY F., MARCHAL E., Façons de recruter : le jugement des compétences sur le marché du travail, Paris, Métailié, 1997.

FRAENKEL B., "La résistible ascension de l'écrit au travail », A. Borzeix \& B. Fraenkel (dir.), Langage et travail : communication, cognition, action, Paris, CNRS, 2001. GHIGLIONE R., L’homme communiquant, Paris, Armand Colin, 1986.

GOFFMAN E., Mise en scène de la vie quotidienne : volume 1, la présentation de soi, Paris, Éditions de Minuit, 1973.

HOTIER H., « La communication internationale des organisations à l'épreuve des cultures nationales et de la culture d'entreprise ", Communication E organisation, 1996, 10.

ISGOUR M., «L'atteinte à l'image de marque des personnes morales », Auteurs E̋ Média, 2007, 3.

KASBARIAN J.-M., BAGGIONI D., GRIMALDI E., Génèse de la (des) norme(s) linguistique(s), Aix-en-Provence, Publications de l'Université de Provence, 1994.

KORCHIA M., Une nouvelle typologie de l'image de marque. Actes du $16^{\mathrm{e}}$ congrès international de l'Association Française du Marketing, Montréal, Canada, 2000.

LANDRAGIN F., "Saillance physique et saillance cognitive ", Cognition, Représentation, Langage, 2004, 2.

LELEU-MERVIEL S., USEILLE Ph., " Quelques révisions du concept d'information ", dans Papy F. (dir.), Problématiques émergentes dans les sciences de l'information, 2008.

MACCIONI H., "Usages et fonctions stratégiques des signes dans l'entreprise dématérialisée ", dans Hannoun Ch. (dir.), La dématérialisation de l'entreprise : essais sur les dimensions immatérielles de l'entreprise, Paris, L'Harmattan, 2010.

MANESSE D., COGIS D., Orthographe : à qui la faute? Paris, ESF, 2007.

MILLET A., BILLIEZ J., " Futurs professeurs, futurs secrétaires, une orthographe professionnelle ?", dans Lucci V. \& Millet A. (dir.), L'orthographe de tous les jours : enquête sur les pratiques orthographiques des Français, Paris, Honoré Champion, 1994.

NUSSENBAUM M., "Évaluation du préjudice de marque : le cas particulier de l'atteinte à l'image de marque », La Semaine Juridique, 50, 1993.

PACH C., JACQUEMIN D., "Usages et normes dans les écrits ordinaires ", dans Lucci V. \& Millet A. (dir.), L'orthographe de tous les jours : enquête sur les pratiques orthographiques des Français, Paris, Honoré Champion, 1994.

PETIT P., DUGUET E., L'HORTYY., du PARQUET L., SARI F., « Discriminations à l'embauche des jeunes Franciliens et intersectionalité du sexe et de l'origine : les résultats d'un testing ", Centre d'Études de l'Emploi, 2011, 141.

ROTTER J., Social learning and clinical psychology, New York, Prentice-Hall, 1954.

STEINER D. D., « Perceptions des méthodes de recrutement en France : la perspective de la justice organisationnelle », Psychologie du Travail et des Organisations, 2000, 6. 
WATZLAWICK P., BEAVIN J. H., JACKSON D. D., Une logique de communication, Paris, Seuil, 1972.

WOLTON D., Penser la communication, Paris, Flammarion, 1997.

WYNANTS B., L'orthographe, une norme sociale, Liège, Mardaga, 1997.

Résumé : Cette contribution interroge les effets discriminants de l'orthographe, au cours d'un processus de recrutement en entreprise, à travers la réaction engendrée par la lecture des documents de candidature. Un dispositif expérimental en milieu naturel est mis en œuvre pour comparer les prises de contact initiées par les recruteurs suite à la réception de deux candidatures à une offre d'emploi : l'une à l'orthographe impeccable, l'autre à l'orthographe lacunaire. L'auteur démontre que la norme graphique agit en tant que critère de sélection à l'embauche.

Mots-Clés : orthographe, discrimination à l'embauche, recrutement, critère de sélection.

Abstract: This paper questions the discriminatory effect of spelling during a recruitment process through the reaction generated by reading the application documents. An experimental device in natural environment is implemented to compare contacts initiated by recruiters after receiving two applications for a job: one with perfect spelling, the other with faulty spelling. The author demonstrates that the spelling standards act as a selection criterion for hiring.

Keywords : Spelling, discrimination in hiring, recruitment, selection criterion. 